\title{
RESPUESTA INMUNE EN NEONATOS BOVINOS DE MADRES VACUNADAS Y NO VACUNADAS CON BACTERINA CONTRA NEUMOENTERITIS
}

\author{
IMMUNE RESPONSE IN CALVES BORN TO COWS \\ VACCINATED OR NOT VACCINATED WITH BACTERIN \\ AGAINST NEUMOENTERITIS
}

\author{
1 María Inés Castrillón Rodríguez, ${ }^{2}$ Rómulo Campos Gaona \\ ${ }^{1}$ MSc, Universidad Nacional de Colombia, Palmira, Colombia \\ ${ }^{2}$ DSc, Universidad Nacional de Colombia, Palmira, Colombia \\ ${ }^{1}$ micastrillonr@unal.edu.co, ${ }^{2}$ rcamposg@unal.edu.co
}

\begin{abstract}
Citación: Castrillón, M., y Campos, R. (2021). Respuesta inmune en neonatos bovinos de madres vacunadas y no vacunadas con bacterina contra neumoenteritis. Revista de Investigación Agraria y Ambiental, 12(2),

99 - 113. DOI: https://doi.org/10.22490/21456453.3620
\end{abstract}

\section{RESUMEN}

Contextualización: la diarrea neonatal es una patología común en terneros, causa alta mortalidad y disminución de la productividad ganadera.

Vacío de investigación: una propuesta para su control es inmunizar las madres contra enteropatógenos, sobre lo cual no se ha realizado suficiente investigación en Colombia.

Propósito del estudio: el objetivo del presente trabajo fue determinar indicadores de inmunidad pasiva en neonatos bovinos, nacidos de vacas vacunadas con una bacterina comercial contra neumoenteritis en el último tercio de la gestación.

Metodología: veintiún vacas gestantes mestizas fueron distribuidas en tres grupos: T1, control; T2, aplicación de una dosis del biológico 30 días preparto y T3 aplicación de dos dosis a los 30 y 15 preparto; y un cuarto grupo,
T4, para evaluar inmunidad activa, fue conformado por neonatos vacunados a las 24 horas de vida. El período experimental para las vacas comprendió entre 30 días preparto hasta el parto; para los terneros entre 24 y 72 horas posteriores al nacimiento. En las crías, se analizaron indicadores del metabolismo proteico e inmunoglobulinas (Ig).

Resultados y conclusiones: en las vacas, se analizó la calidad del calostro, cinco indicadores del metabolismo proteico y el cortisol como modulador inmune. Estos no presentaron diferencias estadísticas significativas entre tratamientos, excepto AST en el T3. En los neonatos la IgA e IgG no presentaron diferencias significativas ni para tratamientos ni para tiempo. Tanto la IgM como la albúmina presentaron significancia entre tratamientos, mientras que globulinas y proteínas totales lo hicieron solo para el efecto tiempo. No se evidenció 
efecto de la bacterina, ni se encontró falla en la transferencia de inmunidad pasiva.

Palabras clave: calostro; inmunoglobulinas; neumoenteritis; terneros; vacunación

\section{ABSTRACT}

Contextualization: Neonatal diarrhea is a common pathology in calves, causing high mortality and decreased livestock productivity.

Knowledge gap: A proposed option for their control is the immunization of dams against enteropathogens, which has not had enough research in Colombia.

Purpose: The objective of this work was to determine indicators of passive immunity in bovine neonates, born of cows vaccinated with commercial bacterin against pneumoenteritis applied in the last third of gestation.

Methodology: Twenty-one crossbred pregnant cows were distributed in three groups: T1, control; T2, application of a dose of the biological 30 days prepartum, and T3 application of two doses at 30 and 15 days prepartum; and a fourth group, T4, for active immunity determination, of neonates vaccinated with bacterin at 24 hours of life. The experimental period for cows ranging from 30 days antepartum until calving, calves between 24 and 72 hours after birth.

Results and conclusions: In the calves, indicators of protein metabolism and immunoglobulins (Ig) were analyzed. Colostrum quality, five indicators of protein metabolism and cortisol as an immune modulator were analyzed in cows, they did not present significant statistical differences between treatments, with the exception of T3 in AST. In neonates IgA and IgG had no significant differences between treatments or for time. IgM and albumin had significant differences for treatments or time, while globulins and total proteins only for time. No effect of bacterin applications was evidenced and no failure was found in the transfer of passive immunity.

Keywords: calves; colostrum; immunoglobulins; vaccination

\section{RESUMEN GRÁFICO}

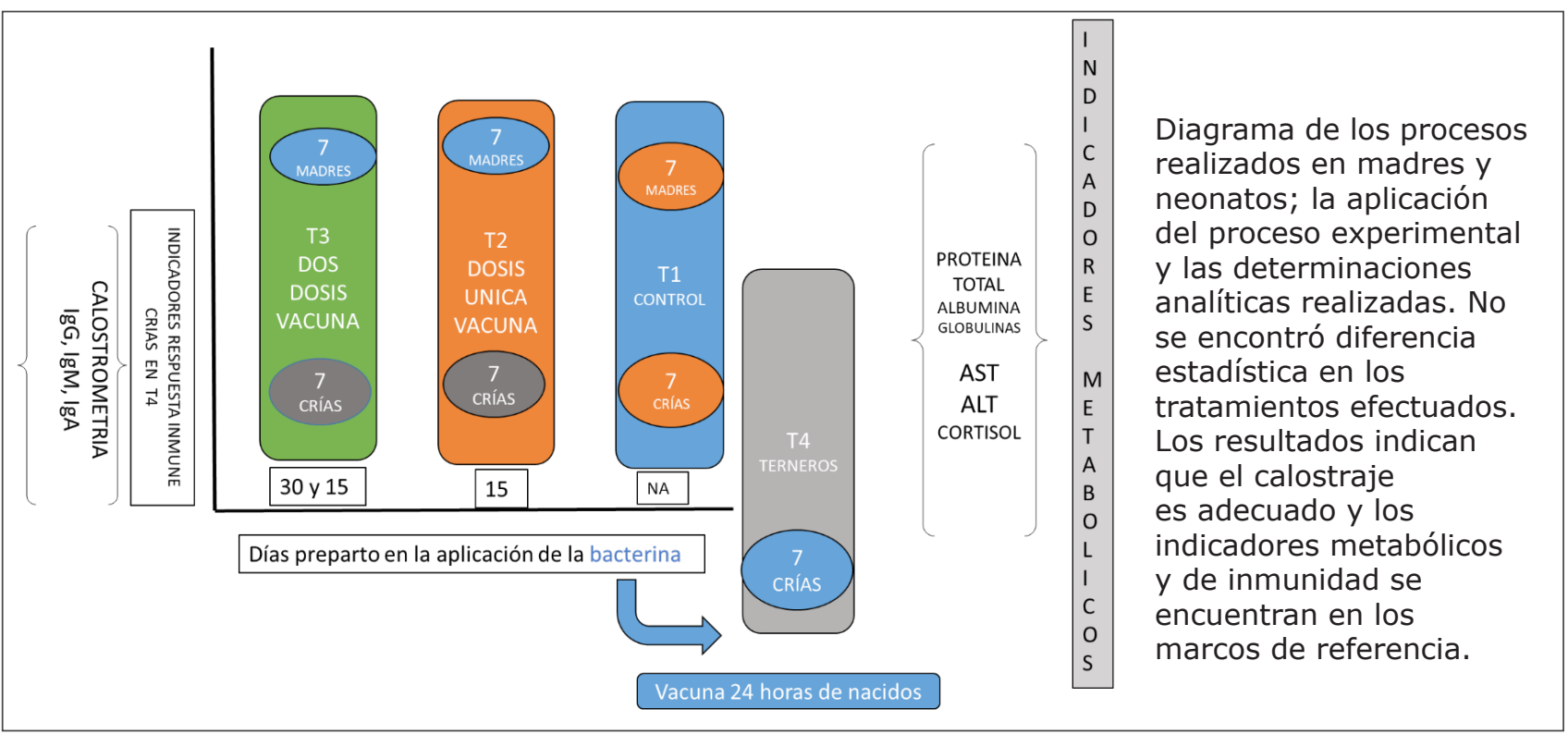




\section{INTRODUCCIÓN}

Una de las principales causas de pérdidas económicas en bovinos es la alta mortalidad neonatal, ocasionada principalmente por diarreas de diverso origen y potenciadas por fallas en la transferencia de inmunidad pasiva (FTIP), lo que origina problemas de rentabilidad, salud pública y de bienestar animal porque ocasionan mayores niveles de morbilidad y mortalidad, largos períodos de cría y mayor uso de antimicrobianos en esta fase de vida (Al-Alo et al., 2018). Se indica que la diarrea neonatal es la patología más común que se presenta en los terneros de menos de 15 días de edad y causa una importante de disminución de la productividad en la industria ganadera. Los terneros nacen sin anticuerpos para su protección, por lo cual dependen de la trasferencia materna de las inmunoglobulinas ( $\mathrm{Ig}$ ) presentes en el calostro, proceso conocido como transferencia de inmunidad pasiva (TIP) (Godden et al., 2019). Sin embargo, los factores maternos asociados con la calidad de la síntesis de Ig inciden en la calidad de estas, siendo necesario conocer a través de indicadores metabólicos algunas posibles causas que afecten al calostro (Sordillo et al., 2009; Mulder et al., 2018).

En Colombia, las altas tasas de morbimortalidad asociadas a diarrea neonatal oscilan entre un $26,1 \%$ y $48 \%$ en la fase de cría (Pardo \& Oliver, 2012). En un estudio de caso se informa una morbilidad del $94,9 \%$ originada en al menos cinco diferentes agentes etiológicos (Cadavid et al., 2014), lo cual indica su importancia para el país. Por otra parte, se menciona que la diarrea neonatal es ocasionada por una infección combinada de diferentes enteropatógenos Sherwin y Down (2018), o por un solo agente, entre los cuales están la Escherichia coli enterotoxigénica cepa F5 (K99) y F41 y la E. Coli
Veteroxigénica (0157:H7) y otros tales como Cryptosporidium parvum, Cryptosporidium muris, Campylobacter jejuni, Campylobacter fecalis, Giardia spp., Salmonella enterica, Serovar Dublin y serovar Typhimurium, que infectan las células epiteliales del intestino ocasionando diarrea. Esto, a su vez, disminuye la absorción de nutrientes esenciales, lleva a pérdida de peso, deshidratación y muerte (Athanasiou et al., 2019; Tóthová et al., 2017; Cadavid et al., 2014).

La inmunidad pasiva se logra de distintas maneras: principalmente, mediante el uso de vacunas que buscan generar memoria inmune para que el sistema inmunitario del animal vacunado, ante un nuevo desafío con el agente o agentes contenidos en la vacuna desarrolle una respuesta inmunológica rápida, específica y de un nivel y duración adecuado que lo proteja adecuadamente (Gamietea, 2019); cuando un neonato bovino consume calostro de calidad y en cantidad, de tal manera que se transfieran inmunoglobulinas que le protegen contra los patógenos (Abdullahoğlu et al., 2019); y la otra posibilidad es que sufra la infección en sí. Igualmente, se conoce que la respuesta inmune mediada por células en animales alimentados con calostro materno que contenía leucocitos, fue significativamente mayor cuando las madres habían sido vacunadas con un antígeno específico, que cuando no lo habían sido (Godden et al., 2019).

Para asegurar la óptima calidad del calostro (>10 mg/mL de IgG) producido por las vacas (Lago et al., 2018), además de la condición nutricional y el estado sanitario de estas, se recomienda la vacunación durante la gestación; se considera que la inmunización aumenta la producción y el paso de anticuerpos maternos al calostro (Abdullahoğlu 
et al., 2019). Asimismo, se ha demostrado que la protección inmune que producen las vacas mediante la aplicación de bacterinas que contienen cepas atenuadas de $E$. Coli se transmite eficientemente a los terneros mediante el consumo de calostro en las primeras horas de vida (Godden et al., 2019). Por esto se ha propuesto que al secado las vacas sean vacunadas con un biológico polivalente que genere anticuerpos maternos que serán transmitidos mediante el calostro a la cría (Sherwin \& Down, 2018).

El objetivo principal de este estudio fue conocer el comportamiento de los indicadores de inmunidad pasiva o activa en terneros que tomaron calostro en las primeras horas de vida, nacidos de madres inoculadas con una bacterina comercial múltiple contra neumoenteritis y en neonatos vacunados pero nacidos de madres que no recibieron la vacuna, en estas se analizaron los metabolitos séricos asociados con su respuesta.

\section{MATERIALES Y MÉTODOS}

Localización: la investigación se realizó en un sistema comercial de producción lechera de tipo semiintensivo, ubicado en trópico bajo $\left(3^{\circ} 49^{\prime} \mathrm{N}, 7^{\circ} 23^{\prime} \mathrm{W}\right)$, ubicado en una formación ecológica de Bosque Seco Tropical (BS-T), con altitud de $950 \mathrm{msnm}$, temperatura promedio de $26^{\circ} \mathrm{C}$, y precipitación media anual de 1000 mm (Holdridge, 1978).

\section{Unidades experimentales y tratamien-} tos: el experimento fue avalado institucionalmente por el comité de ética de investigación animal (CP-FCA Acta 03-2015). Se utilizaron 21 vacas mestizas ( $F 1$, Gyr x Holstein) entre tercer y cuarto parto, en el octavo mes de gestación. Los animales se dividieron en tres grupos, cada uno con siete vacas y sus respectivas crías al nacimiento. Se conformó un cuarto grupo con siete crías recién nacidas. Los tratamientos fueron: control (T1); tratamiento 2 (T2), aplicación de una dosis de 2 $\mathrm{ml}$ de bacterina comercial contra neumoenteritis 30 días antes del parto; tratamiento 3 (T3), aplicación de bacterina comercial contra neumoenteritis, 30 días antes del parto, y 15 días preparto; tratamiento 4 (T4), aplicación de una dosis a terneros a las 24 horas de vida. La bacterina contenía Salmonella dublin 25\%, Salmonella typhimurium 25\%, Escherichia coli K99 25\%, Pasteurella multocida tipo II $20 \%$, inactivante, vehículo tipo gel $5 \%$ y se aplicó por vía subcutánea.

Manejo animal: las vacas se encontraban en un sistema de pastoreo rotacional con pasto estrella (Cynodon plectostachyus) y suplementación con ensilaje de maíz; posterior al parto se dejaban con la cría las primeras 24 horas donde los terneros tomaban calostro a voluntad, al segundo día se separaban de sus madres y el suministro de calostro se realizaba dos veces al día por medio de biberón a razón de dos litros en cada toma durante tres días, luego pasaban al sistema de crianza de balde estaca hasta el destete.

Recolección muestras de campo: 24 horas después del parto se recolectaron muestras de calostro $(300 \mathrm{ml})$ y sangre en tubos al vacío sin anticoagulante, mediante venipunción coccígea. Las muestras se centrifugaron a 2500 rpm durante 15 minutos, se extrajo el suero y se almacenó a $-20^{\circ} \mathrm{C}$ hasta el análisis de AST, ALT, proteínas totales (PT), globulinas totales, albúmina y cortisol. 
En los terneros, se colectó sangre por venipunción yugular a las $24 \pm 6,48$ y 72 horas posteriores al nacimiento. Se extrajo suero por centrifugación, en la que se analizaron proteínas totales, globulinas totales y albúmina mediante método enzimático colorimétrico y analizador químico semiautomático de lectura óptica RAYTO $^{\circledR}$ (Shenzhen, China), las inmuglobulinas G, M y A (Triple J Farms ${ }^{\circledR}$ ) mediante la técnica de inmunodifusión radial simple y lectura óptica con calibrador. Las muestras de calostro fueron analizadas in situ a $22^{\circ} \mathrm{C}$ con un calostrómetro tipo lactodensímetro, calibrado para lectura en $\mathrm{g} / \mathrm{L}$.

Análisis estadístico: Los datos fueron almacenados en Excel y procesados en el paquete estadístico InfoStat, versión 2017 (Di Rienzo et al., 2017).

El diseño estadístico se basó en un modelo completamente al azar, en el cual una vez evaluada la normalidad y homocedasticidad de la variable se realizó un análisis de varianza de dos vías (tratamientos y tiempo -horas- de respuesta pos nacimiento), las variables dependientes fueron los metabolitos y las Ig y, las variables independientes fueron los tratamientos (vacunaciones). Se aceptó significancia estadística cuando $p<0,05$. Para analizar las diferencias significativas se utilizó la prueba de Duncan y para evaluar las similitudes entre los resultados de las pruebas se utilizó el coeficiente de correlación de Pearson. Se realizó Estadística descriptiva de metabolitos e Ig para los tratamientos y tiempo de muestreo.

\section{RESULTADOS Y DISCUSIÓN}

El consumo de calostro tuvo un efecto significativo para el tiempo de muestreo después del nacimiento y para la concentración de Ig y PT en todos los tratamientos (Figura 1a). Según los conceptos sobre fallas en la transferencia de inmunidad pasiva (FTIP), el calostro es un indicador indirecto de inmunidad pasiva (Godden et al., 2019), se considera que esta existe cuando la concentración de proteínas totales es $\leq 55 \mathrm{~g} / \mathrm{L}$ (Lawrence et al., 2017) o valores de $\leq 52 \mathrm{~g} / \mathrm{L}$ (Cuttance et al., 2017). En este trabajo para ninguno de los tratamientos se encontraron bajas concentraciones de proteínas en calostro, que pudieran asociarse a FTIP, lo que evidencia que los terneros del experimento lograron un buen calostraje.

La respuesta inmune pasiva se relaciona con la calidad de calostro, los valores en este estudio indican que el T2 presentó la concentración calostral de IgG más alta $(85,71$ $\mathrm{mg} / \mathrm{mL})$ seguido por $\mathrm{T} 1(82,86 \mathrm{mg} / \mathrm{mL})$ y $\mathrm{T} 3$ $(61,43 \mathrm{mg} / \mathrm{mL}$ ) (Figura 1d), sin encontrarse diferencia estadísticamente significativa entre tratamientos.

La investigación realizada por Soriano (2014), indica que la aplicación de una bacterina dos meses antes del parto mejora la calidad del calostro, encontrando valores de $52 \mathrm{mg} / \mathrm{mL}$ de IgG calostral; sin embargo, Yepes y Prieto, (2011), informan $57,6 \mathrm{mg} / \mathrm{mL}$ de IgG $\sin$ vacunación, lo cual puede explicarse por variaciones de respuesta al medio ambiente o a factores nutricionales.

En la Figura 1, se presentan los resultados de los metabolitos proteicos ( $1 \mathrm{a}, 1 \mathrm{~b}, \mathrm{y} 1 \mathrm{c})$ y las inmunoglobulinas (1d, 1e y $1 \mathrm{f})$, en los tres periodos posteriores al nacimiento analizados en los neonatos. 
a. Proteina total

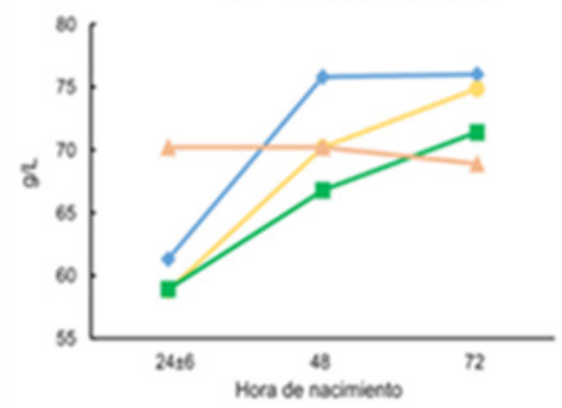

c. Globulinas

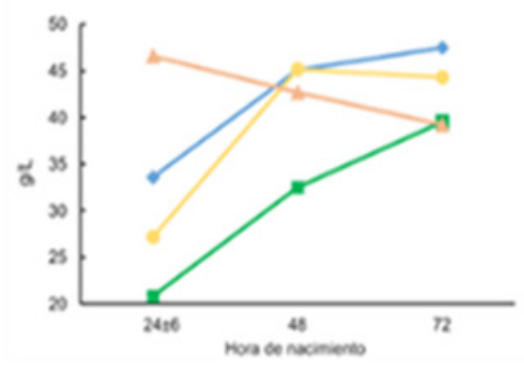

e. $\lg G$

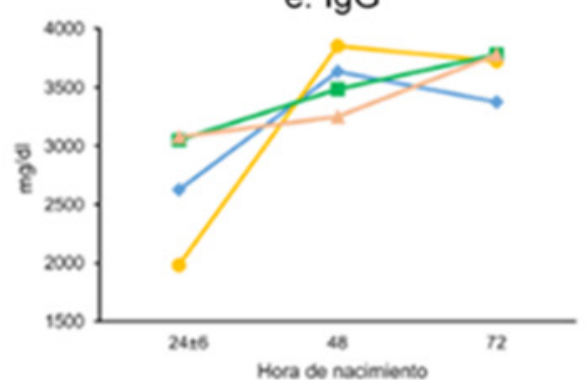

b. Albúmina

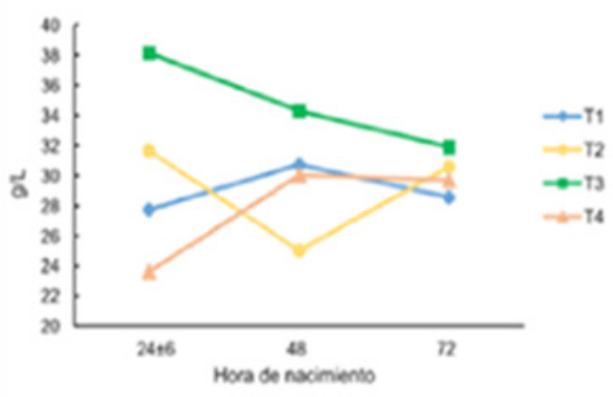

d. IgA

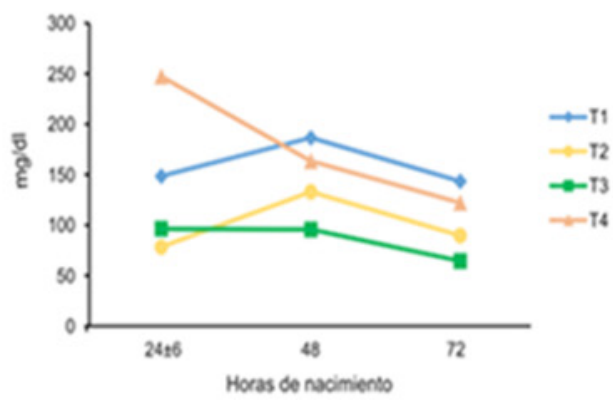

f. $\lg M$

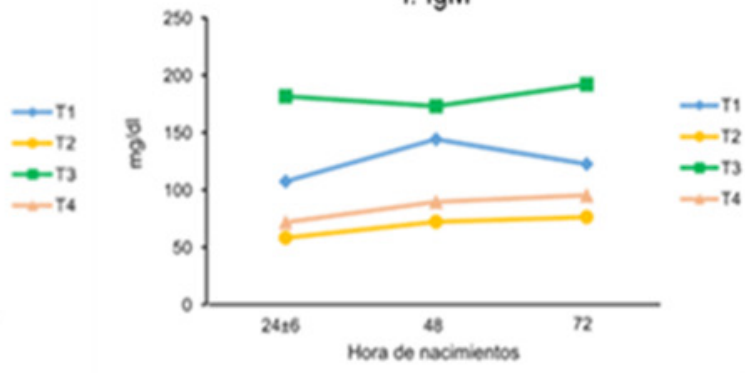

Figura 1. Diagrama de la variación de los metabolitos proteicos a. proteína total, b. albúmina, c. globulina y de las inmunoglobulinas d. IgA, e. IgG y f. IgM, en los neonatos bovinos de cuatro grupos experimentales, control (T1) y respuesta posvacunal (T2, T3 y T4), a las 24,48 y 72 horas posparto.

Fuente: Autores

Las globulinas presentaron menor valor en T3 $(20,79 \mathrm{~g} / \mathrm{L})$ a las $24 \pm 6$ horas de nacimiento, probablemente como producto del bloqueo inmunológico y menor transferencia de Ig al calostro (Windeyer \& Gamsjäger, 2019). El valor más alto $(47,46 \mathrm{~g} / \mathrm{L})$ se presentó en T1 a las 72 horas, lo cual muestra que el consumo de calostro elevó las Ig, independiente de la vacunación. T4 mostró a las $24 \pm 6$ horas mayor concentración en comparación con los demás tratamientos, posiblemente derivado de un mayor consumo de calostro en este grupo. En este estudio se encontraron diferencias significativas en el tiempo para muestras colectadas entre $24 \pm 6$ y 72 horas post nacimiento, a mayor tiempo en el muestreo, concentraciones más elevadas. De Paula y colaboradores (2019) reportan valores entre 24 y $39 \mathrm{~g} / \mathrm{L}$ en terneros durante la primera semana de vida. Asimismo, debe tenerse en cuenta que entre más tiempo posparto pase, menor será la cantidad de globulinas que el ternero pueda 
absorber, debido a la pérdida de absorción de inmunoglobulinas en la mucosa intestinal $y$, por tanto, su concentración tiende a estabilizarse como se muestra e la Figura 1c, para 48 y 72 horas (Godden et al., 2019).

La concentración de inmunoglobulinas IgG (Figura 1e), e IgA (Figura 1d) no presentaron diferencias significativas ni para tiempo, ni entre tratamientos, mientras que IgM (Figura 1f) presentó diferencias significativas entre tratamientos ( $p<0,002)$, hallazgo importante ya que esta Ig brinda mayor protección en las primeras horas de vida (Ježek et al., 2012; Chase et al., 2008).

La concentración de IgG registró el menor valor en T2 (tratamiento con una dosis de vacuna) con $19,8 \mathrm{mg} / \mathrm{mL}$ a las $24 \pm 6 \mathrm{~h}$, y $38,51 \mathrm{mg} /$ $\mathrm{mL}$ a las 48 horas. Los rangos de referencia establecidos por Hampe y Wehrend (2019) están entre 6,7 y $29,0 \mathrm{mg} / \mathrm{mL}$ para terneros en la primer semana de vida. Los valores hallados en los tratamientos son más elevados que otros reportados, posiblemente debido al método analítico empleado en la determinación, dado que la técnica de inmunodifusión radial simple (RID) es más sensible y precisa que la técnica ELISA (Dunn et al., 2018; Gelsinger et al., 2015). Igualmente, se encontró a las $24 \pm 6$ horas de nacimiento valores de IgG superiores a $10,0 \mathrm{mg} / \mathrm{mL}$ en todos los tratamientos, lo cual indica ausencia de FTIP, y se corrobora que la IgG posee la mayor concentración entre las Ig en terneros (Godden et al., 2019).

Respecto a la concentración de Ig pos nacimiento, la concentración de IgA a las $24 \pm 6$ muestra su mayor concentración para T4 con $2,47 \mathrm{mg} / \mathrm{mL}$, tal vez asociada al tipo de antígeno aplicado (Chase et al., 2008), mientras que a las 72 horas, todos los tratamientos presentaron valores bajos, siendo el menor T3 con $0,64 \mathrm{mg} / \mathrm{mL}$. Los valores reportados para IgA están entre 0,5 a 4,5 mg/mL (Kaneko et al., 2008). Para la IgM se encontró el menor valor $(0,58 \mathrm{mg} / \mathrm{mL})$ en $\mathrm{T} 2$ a las $24 \pm 6$ horas de nacimiento mientras que la mayor concentración fue $1,92 \mathrm{mg} / \mathrm{mL}$ a las 72 horas para el T3, el cual recibió dos dosis de vacuna. Se presentó diferencia estadística entre los tratamientos.

Respecto a la concentración de Ig pos nacimiento, la concentración de IgA a las $24 \pm 6$ muestra su mayor concentración para T4 con $2,47 \mathrm{mg} / \mathrm{mL}$, tal vez asociada al tipo de antígeno aplicado (Chase et al., 2008), mientras que a las 72 horas, todos los tratamientos presentaron valores bajos, siendo el menor T3 con $0,64 \mathrm{mg} / \mathrm{mL}$. Los valores reportados para IgA están entre 0,5 a 4,5 mg/mL (Kaneko et al., 2008). Para la IgM se encontró el menor valor $(0,58 \mathrm{mg} / \mathrm{mL})$ en $\mathrm{T} 2$ a las $24 \pm 6$ horas de nacimiento mientras que la mayor concentración fue $1,92 \mathrm{mg} / \mathrm{mL}$ a las 72 horas para el T3, el cual recibió dos dosis de vacuna. Se presentó diferencia estadística entre los tratamientos.

La diferencia en la concentración encontrada para IgG no se puede explicar únicamente como respuesta al proceso vacunal, ya que pudieron variar las condiciones medioambientales en las que permaneció el grupo experimental (Gamietea, 2019; Ježek et al., 2012). Igualmente, debe considerarse la posible estimulación antigénica derivada de patógenos externos a los cuales han podido estar expuestos los terneros; además, los valores en T2 y T4 también fueron más altos a las 72 horas de nacidos, esto no se pudo derivar únicamente del consumo del calostro, sino también, en parte, por el proceso vacunal (Dudek et al., 2017; Uetake et al., 2014).

En los terneros de los cuatro grupos experimentales se encontraron correlaciones significativas $(p<0,0001)$ entre globulinas $e$ $\operatorname{IgG}(0,76)$, con $\operatorname{IgA}(0,66)$ y con $\operatorname{IgM}(0,47)$ 
y, entre globulinas y proteínas totales $(0,95)$ este comportamiento de alta relación demuestra lo planteado como evaluación directa e indirecta de inmunidad pasiva, cuando se analizan Ig o PT sérica o con las proteínas totales del calostro (Cuttance et al., 2017; Ježek et al., 2012).

La concentración de proteínas séricas e inmunoglobulinas en los bovinos es baja al nacimiento debido a su sistema placentario y aumenta en el tiempo con el consumo de calostro (Tóthová et al., 2017). En el posparto, la concentración de proteínas séricas en el neonato está relacionada con el consumo de calostro y la calidad del mismo, lo cual explica las altas concentraciones a las 48 y 72 horas de nacimiento (Figura 1a), tal como se evidencia para el grupo control. La concentración de proteína total (PT) y globulinas, en terneros presentó una diferencia significativa en el efecto tiempo pos nacimiento; para el efecto tratamiento solo hubo efecto significativo para la albúmina; tampoco se presentó interacción tiempo*tratamiento para los metabolitos proteicos.

El menor valor de PT se registró en T2 a las $24 \pm 6$ horas con 58,93 g/L y el T1 presentó la mayor concentración a las 48 y 72 horas con 75,8 y $76,01 \mathrm{~g} / \mathrm{l}$ respectivamente. Klinkon y Ježek (2012) registraron valores de $45,8 \mathrm{~g} / \mathrm{L}$ en terneros después del nacimiento, $54,5 \mathrm{~g} / \mathrm{L}$ después de la ingesta de calostro y $50-70 \mathrm{~g} / \mathrm{L}$ al mes de edad. Las mayores concentraciones de $\mathrm{PT}$, se presentan después de consumir calostro, esto asociado con las inmunoglobulinas que son su mayor componente (Godden et al., 2019).

La mayor concentración de albúmina (38,13 $\mathrm{g} / \mathrm{L})$ se registró en T3 y el valor más bajo $(23,61 \mathrm{~g} / \mathrm{L})$ en T4 a las $24 \pm 6$ horas de nacimiento $(p<0,05)$ como se presenta en la Figura $1 b$; a mayor concentración de globulinas menor concentración de albúmina. Hampe y Wehrend, (2019) informan que la albúmina en terneros puede estar entre 19 y $33 \mathrm{~g} / \mathrm{L}$. En este estudio, los valores son superiores a los descritos y se encontró efecto significativo de la vacunación, posiblemente debido a las características de la bacterina, según lo mencionan Petzl et al., (2018), esta puede activar las moléculas co-estimuladoras (CD80, CD86 y CD40), que a su vez inducen a la células presentadoras de antígeno (APC) a producir interleucinas, que intervienen en la respuesta inflamatoria (Tóthová et al., 2017), que produce mayor permeabilidad de la membrana. Esto permite el paso de albúmina a través del mismo receptor de IgG, como también sucede cuando se inmuniza con antígenos bacteriales (Dudek et al., 2017).

Cuando el calostro tiene una mayor concentración de albúmina, esta puede pasar al ternero mediante el mismo receptor (FcRn) de IgG (Stark et al., 2015). Se ha demostrado que el FcRn se une a la albúmina, la cual es ocho veces más abundante en el calostro y, al unirse al receptor, lo hace en un emplazamiento distinto y no competitivo con el de IgG, lo cual explicaría la concentración de albúmina en los terneros del T3 (Guzmán y Olivera-Angel, 2020).

En las vacas, las concentraciones séricas de proteína total, albúmina y globulina, analizadas como indicadores indirectos del metabolismo proteico (Sordillo et al., 2009), no presentaron diferencia significativa entre tratamientos. Los valores de PT registrados son de 67,75 y $80,83 \mathrm{~g} / \mathrm{L}$ para T3 y T2, respectivamente. Los valores de referencia están entre 67,4 y 74,6 g/L (Kaneko et al., 2008). En este estudio, T2 presentó un valor superior a la media de referencia, derivado de la mayor concentración de globulinas (Tabla 1 ). Roa-Vega y colaboradores (2017), reportan 
concentraciones de 79,3 g/L, siendo estos valores similares a los encontrados por Guerra et al. (2018) y a los hallados en el presente experimento, lo cual evidencia el buen manejo nutricional en la explotación. Los valores informados indican una alta concentración sérica de proteína, asociada en parte al aumento de las globulinas por el proceso experimental de vacunaciones en T2 y T3.

En vacas, a mayor número de partos mayores valores séricos de proteína total, debido principalmente a las inmunoglobulinas, ya que el sistema inmunológico de las vacas multíparas ha estado en contacto con más patógenos, y por lo tanto tienen una mayor cantidad de anticuerpos (Bobbo et al., 2017). Las diferencias entre tratamientos (Tabla 1) pueden estar relacionadas con la respuesta inmune a la bacterina; no obstante, T2 recibió una dosis y T3 dos, mostrando este, contradictoriamente, una menor concentración de globulinas, probablemente por bloqueo materno a la respuesta exógena inducida (Windeyer y Gamsjäger, 2019).

Tabla 1. Medias y análisis de varianza para las concentraciones séricas de metabolitos proteicos en vacas sometidas a diferentes ritmos de aplicación de una bacterina contra neumoenteritis.

\begin{tabular}{l|c|c|c|c|c}
\multirow{2}{*}{ Variable (g/L) } & \multicolumn{3}{c}{ Tratamientos } & f & p-valor \\
\cline { 2 - 5 } & T1 & T2 & T3 & 3,50 & 0,0519 \\
\hline Proteína total & $77,62^{a}$ & $80,83^{a}$ & $67,75^{a}$ & 0,67 & 0,5241 \\
\hline Albúmina & $30,43^{a}$ & $32,51^{a}$ & $30,08^{a}$ & 2,82 & 0,0860 \\
\hline Globulina & $47,19^{a}$ & $48,32^{a}$ & $37,67^{a}$ & 2 & 2 \\
\hline
\end{tabular}

Cuando $\mathrm{p}<0,05$ existen diferencias estadísticas.

Fuente: autores

La albúmina presentó para T2 la mayor concentración con 32,51 g/L (Tabla 1 ). Kaneko et al., (2008) indican valores para este metabolito entre 30,3 y 35,5 g/L. Según Da Silva, et al. (2019), la albúmina puede variar en función del estado fisiológico, el clima y la alimentación. Al final del periodo de gestación, la albúmina es baja debido a que es catabolizada para aportar aminoácidos necesarios para la producción de globulinas (Tóthová et al., 2017). Debido a que no se encontraron diferencias entre tratamientos (Tabla 1), la aplicación de bacterinas contra la neumoenteritis en el último mes de gestación no afecta significativamente este metabolito.
Las globulinas séricas en las madres mostraron el valor más bajo en T3 con 37,67 g/L, mientras que en $\mathrm{T} 2$ se presentó el mayor con $48,32 \mathrm{~g} / \mathrm{L}$; los valores conocidos están entre 30 y 34,8 g/L (Kaneko et al., 2008). Los valores hallados en los tratamientos con vacuna son superiores (Tabla 1) al valor del grupo control, pudiendo indicar efecto de la bacterina; sin embargo, el T3 que recibió dos dosis de vacuna, presentó un valor más bajo que T2 posiblemente por inhibición asociada al bloqueo materno (Windeyer y Gamsjäger, 2019). En general, Roa-Vega et al., (2017) informan valores de 42,86 33,47 para globulinas en vacas recién paridas con suplementación 
proteica. Estos valores son más cercanos a los del presente trabajo; mientras que, en condiciones similares a las de este experimento para tipo racial y únicamente durante el período de transición se encontraron valores cercanos a $30 \mathrm{~g} / \mathrm{L}$ (Campos et al., 2012), lo que indica las amplias variaciones en los valores de globulinas, los desafíos medioambientales y el posible efecto de la vacuna.

Se describe que las globulinas se elevan debido a enfermedades o vacunaciones recientes (Tizard y Payne, 2019). Así, los valores de globulinas encontrados en T2 se pueden asociar con la estimulación inmunogénica generada por la bacterina, sin embargo, en T3 los bajos valores indicarían que el refuerzo en la inmunización requiere un protocolo de aplicación más amplio entre dosis, para generar una mayor cantidad de anticuerpos transferibles al calostro, para no generar el mencionado bloqueo materno (Gamietea, 2019; Windeyer y Gamsjäger, 2019).

De acuerdo con la práctica común de vacunación realizada por los productores y replicada experimentalmente en el presente trabajo, es posible que la frecuencia de aplicación de la bacterina y el intervalo de tiempo entre aplicaciones haya generado inhibición de la respuesta inmune medida (Windeyer y Gamsjäger, 2019). Diversos autores recomiendan mínimo cuatro semanas de intervalo entre la aplicación de dosis de bacterinas y sugieren empezar la primera dosis al séptimo mes de gestación o al inicio del periodo seco (Sears et al., 2017). No siempre se muestran mejores respuestas con refuerzos posteriores, cuando el intervalo entre dosis es de 15 días o menos, ya que se puede neutralizar la respuesta secundaria (Gamietea, 2019).

En este experimento, al no encontrar diferencias entre tratamientos, no podría deducirse que no hay diferencia entre inmunizar o no inmunizar con bacterinas durante el preparto, dado que el tiempo entre las dosis fue de 15 días o la aplicación única se realizó 30 días antes del parto esperado. Esta es una práctica rutinaria en campo, que posiblemente no permite una respuesta biológica integral por parte de la vacuna (Gamietea, 2019).

Por otra parte, debe tenerse en cuenta el carácter de inactivado del biológico que se aplicó, razón por la cual el fabricante recomienda la doble dosis, al igual que para otros biológicos que incluyan patógenos muertos, vivos atenuados o modificados en los que, en la mayoría de los casos, se emplean dos dosis, con un amplio intervalo de tiempo entre estas (Dudek et al., 2017). Se han encontrado infecciones congénitas para IBR, DVB y Leptospira en crías con FTIP (Lawrence et al., 2017), sin que las vacunas logren inmunizar completamente contra estos patógenos.

Las madres de los terneros del T4, no fueron vacunadas en ningún momento, sus crías recibieron una dosis del biológico y estas no presentaron diferencias en la síntesis de calostro con relación a las madres de los terneros nacidos de madres inmunizadas.

Vacunar cuando existen anticuerpos maternos circulantes se considera un problema de protección inmune. Esta situación se conoce como anticuerpos en fase materna (In face of maternal antibody, IFOMA, por sus siglas en inglés) (Chamorro et al., 2016). Esto pudo impedir observar diferencias entre inmunidad pasiva y activa, porque la vacuna muerta no pudo inducir una respuesta humoral dado que existían anticuerpos maternos; asimismo, es necesario tener en cuenta el bloqueo al desarrollo de inmunidad en una cría con alta protección calostral, como sucede en los mamíferos nacidos de placentación sindesmocorial 
(Windeyer y Gamsjäger, 2019), lo cual explicaría la ausencia de diferencias para el T4, que recibió una dosis vacunal en una edad en la cual no hay respuesta del sistema inmune (Sherwin y Down, 2018).

Se analizaron ALT y AST en las vacas para monitorear la función hepática y el cortisol como posible responsable de inmunosupresión en el periparto. Ninguno presentó diferencias significativas para tratamientos (Tabla 2), lo cual indica que las madres estuvieron bajo condiciones similares de salud hepática y nivel de estrés (Uetake et al., 2014).

La concentración de ALT hallada en los tratamientos está dentro de los valores de referencia (7 a $35 \mathrm{U} / \mathrm{L}$ ) mencionados por Roa-vega et al., (2017). La ALT sérica se incrementa por alteración en la membrana del hepatocito, haciéndolo más permeable y permitiendo la liberación de la enzima al torrente sanguíneo (Dharmayudha et al., 2018).

La concentración de AST presentó diferencias significativas entre tratamientos (Tabla 2). Los valores hallados son normales de acuerdo a lo citado por Kaneko et al., (2008). La diferencia significativa encontrada en esta investigación puede explicarse mediante el alto valor de AST en T2 y T3, lo cual puede estar asociado con la concentración de cortisol debido al estrés causado por el manejo de aplicación de la vacuna, tal como se reporta en investigaciones realizadas por Shawky y colaboradores (2016) y El-Mandrawy y Farag (2017), quienes también aplicaron vacunas y hallaron niveles altos de AST, tal vez por procesos inflamatorios inespecíficos postvacunales, lo que asociaron con altas concentraciones de cortisol derivadas del estrés del parto y la vacunación.

Tabla 2. Medias y análisis de varianza de las concentraciones séricas de ALT y AST enzimas indicadoras de función hepática, cortisol y Calostrometría en vacas sometidas a una o dos aplicaciones de una bacterina contra neumoenteritis.

\begin{tabular}{l|c|c|c|c|c}
\hline \multirow{2}{*}{ VARIABLE } & \multicolumn{3}{c}{ Tratamientos } & f & p-valor \\
\cline { 2 - 5 } & T1 & T2 & T3 & 1,54 & 0,2415 \\
\hline $\begin{array}{l}\text { Alanina Aminotransferasa } \\
\text {-ALT (U/L) }\end{array}$ & $17,82^{\mathrm{a}}$ & $20,00^{\mathrm{a}}$ & $21,70^{\mathrm{a}}$ & & 0,0013 \\
\hline $\begin{array}{l}\text { Aspartato aminotransferasa } \\
\text { AST(U/L) }\end{array}$ & $103,74^{\mathrm{a}}$ & $137,95^{\mathrm{b}}$ & $115,25^{\mathrm{a}}$ & 9,76 & 0,5181 \\
\hline Cortisol (nmol/L) & $191,86^{\mathrm{a}}$ & $180,38^{\mathrm{a}}$ & $253,73^{\mathrm{a}}$ & 0,68 & 0,334 \\
\hline Calostrometría (mg/mL) & $82,86^{\mathrm{a}}$ & $85,71^{\mathrm{a}}$ & $61,43^{\mathrm{a}}$ & 1,17 & 0 \\
\hline
\end{tabular}

Cuando $\mathrm{p}<0,05$ se considera que existen diferencias estadísticas.

Fuente: Autores

Las concentraciones de cortisol en este estudio son similares a las mencionados por Shawky et al., (2016) en vacas recién paridas; sin embargo, en T3 fue considerablemente más alta, lo cual se atribuye posiblemente al manejo derivado de las dos dosis de vacuna que se aplicaron, con estrecho intervalo de 15 días, lo que pudo generar más estrés y mayor concentración de cortisol, como se ha evidenciado en otros trabajos con manipulaciones en animales durante el preparto (Uetake et al., 2014). 
La calostrometría para evaluar la síntesis de calostro en las madres vacunadas o no, se presenta en la Tabla 2, donde se muestran valores correspondientes a calostro de excelente calidad en todos los tratamientos. Los estándares referenciados son: menor a $20 \mathrm{~g} / \mathrm{L}$, para mala calidad; 20 a $50 \mathrm{~g} / \mathrm{L}$, calidad moderada y superior a $50 \mathrm{~g} / \mathrm{L}$ para excelente calidad (Lago et al., 2018). El grupo control, al no mostrar diferencias con T2, indica que en todas las madres el calostro fue sintetizado en forma oportuna y brindó al neonato adecuada protección. Todas las vacas generaron respuesta a los desafíos medioambientales o a vacunaciones previas, aún el T3, con una concentración baja con relación a los grupos experimentales, pero superior a lo esperado en calostros de calidad, permitió inferir en todos los tratamientos una adecuada síntesis de calostro, independiente de la vacunación. Existen diversas circunstancias asociadas a la cantidad y frecuencia en el suministro de calostro que pueden originar variaciones en la respuesta; sin embargo, estas no fueron objetivo del presente trabajo.

\section{CONCLUSIONES}

Se encontraron diferencias estadísticas para IgM únicamente entre los indicadores de inmunidad activa o pasiva de neonatos nacidos de vacas inmunizadas o no, o en los terneros inmunizados a las 24 horas posteriores al nacimiento, siguiendo el esquema vacunal propuesto en este trabajo, probablemente por el bloqueo de inmunidad activa por parte de la cría y por el proceso de respuesta descrito como IFOMA.

La concentración de proteína total (PT) y globulinas en terneros presentó diferencia significativa para el efecto del tiempo de evaluación posterior al nacimiento, asociado al factor acumulativo del consumo de calostro posparto.

En las vacas, la PT, la albúmina y las globulinas no presentaron diferencias, por lo tanto, la aplicación de bacterina contra neumoenteritis, según el protocolo propuesto en esta investigación, no generó ningún efecto en estos metabolitos.

La concentración de cortisol no afectó la respuesta en ninguno de los tratamientos.

\section{CONTRIBUCIÓN DE LA AUTORÍA}

Primer autor: investigación, análisis de datos, escritura. Segundo autor: conceptualización, análisis de datos, escritura, revisión y edición.

\section{AGRADECIMIENTOS}

Los autores agradecen a la vicerrectoría de Investigación de la Universidad Nacional de Colombia, la financiación del presente trabajo y, a los propietarios y personal de la hacienda Chiquique (Yotoco, Valle del Cauca) la logística de campo para el desarrollo del experimento.

\section{LITERATURA CITADA}

Abdullahoğlu, E., Duru, S., Özlüer, A., y Filya, İ. (2019). Factors affecting colostrum quality and calf passive transfer levels in Holstein cattle. Animal Science Papers \& Reports, 37(1):2939. Recuperado de: http://a192.fsi.pl/year-2019vol-37-no-1

Al-Alo, K. Z. K., Nikbakht Brujeni, G., Lotfollahzadeh, S., Moosakhani, F., \& Gharabaghi, A. (2018). Correlation between neonatal calf diarrhea and the level of maternally derived antibodies. Iranian Journal of Veterinary Research, 19(1), 3-8. Recuperado de: https://www.ncbi.nlm.nih.gov/ pmc/articles/PMC5960765/

Athanasiou, L. V., Katsoulos, P. D., Ziogas, C., Kassab, A., y Polizopoulou, Z. (2019). Serum protein electrophoretic profile in diarrheic neonatal calves. Comparative Clinical Pathology (2019), 28, 685-688. https://doi.org/10.1007/s00580-01902935-w

Bobbo, T., Fiore, E., Gianesella, M., Morgante, M., Gallo, L., Ruegg, P. L., ... Cecchinato, A. (2017). Variation in blood serum proteins and association with somatic cell count in dairy cattle from multi-breed herds. animal, 11(12), 2309-2319. https://doi.org/10.1017/S1751731117001227 
Cadavid, D. A., Giraldo, C. A., Sierra, S., Montoya, M., Chaparro, J. J., Restrepo, J. E., y OliveraÁngel, M. (2014). Diarrea neonatal bovina en un hato del altiplano norte de Antioquia (Colombia), un estudio descriptivo. Veterinaria y Zootecnia. 8(2): 120-129. http://bibliotecadigital.udea.edu. co/handle/10495/10947

Campos, R., García, K., Hernández, E. A., y Giraldo, L. (2012). Protein and mineral metabolites for dairy cows during the transition period under tropical conditions. Revista Facultad Nacional de Agronomía Medellín, 65(2), 6719-6728.

Chamorro, M. F., Woolums, A., y Walz, P. H. (2016). Vaccination of calves against common respiratory viruses in the face of maternally derived antibodies (IFOMA). Animal health research reviews, 17(2), 79-84. https://doi.org/10.1017/ $\underline{\mathrm{S} 1466252316000013}$

Chase, C. C., Hurley, D. J., y Reber, A. J. (2008). Neonatal immune development in the calf and its impact on vaccine response. Veterinary Clinics of North America: Food Animal Practice, 24(1), 87104. https://doi.org/10.1016/j.cvfa.2007.11.001

Cuttance, E. L., Mason, W. A., Denholm, K. S., Laven, R. A. (2017). Comparison of diagnostic tests for determining the prevalence of failure of passive transfer in New Zealand dairy calves. New Zealand Veterinary Journal, 65(1), 6-13. https:// doi.org/10.1080/00480169.2016.1230525

Dharmayudha, A. A. G. O., Kusumadarma, I. B. D., Ardana, I. B. K., Anthara, M. S., Gunawan, I. W. N. F., Sudimartini, L. M., y Agustina, K. K. (2018). Aktivitas Alanin Aminotransferase Dan Aspartat Aminotransferase Sapi Bali Terinfeksi Fasciola Gigantica. Buletin Veteriner Udayana, 10(1), 87-92. https://doi.org/10.24843/bulvet.2018. v10.i01.p14

Da Silva, M. S. C., dos Santos Rodrigues, D., Tuerlinckx, S., y Siqueira, C. M. G. (2019). Perfil bioquimico de Albúmina e Colesterol em vacas de invernar submetidas a suplementação. Anais do Salão Internacional de Ensino, Pesquisa e Extensão, 10(1). Recuperado de: http://200.132.146.161/index.php/siepe/article/ view/38760/23576

De Paula, Marília Ribeiro, Slanzon, Giovana Simão, Sobreira, Natália, \& Bittar, Carla Maris Machado. (2019). Passive transfer of immunity in dairy calves with additional consumption of immunoglobulin through colostrum supplement: effects in health and performance. Revista Brasileira de
Saúde e Produção Animal, 20, e0242019. Epub May 02, 2019. https://doi.org/10.1590/s15199940200242019

Di Rienzo, J. A., Casanoves, F., Balzarini, M. G., Gonzalez, L. A., y Tablada, E. M. (2017). InfoStat, versión 2017. Universidad Nacional de Córdoba, Argentina. Recuperado de http://www.infostat.com.ar/index.php?mod=page\&amp;id=34

Dudek, K., y Bednarek, D. (2017). T-and B-cell response analysis following calf immunisation with experimental Mycoplasma bovis vaccine containing saponin and lysozyme dimer. Journal of Veterinary Research, 61(4), 433-437. https://doi. org/10.1515/jvetres-2017-0060

Dunn, A., Duffy, C., Gordon, A., Morrison, S., Argüello, A., Welsh, M., y Earley, B. (2018). Comparison of single radial immunodiffusion and ELISA for the quantification of immunoglobulin $\mathrm{G}$ in bovine colostrum, milk and calf sera. Journal of Applied Animal Research, 46(1), 758-765. https://doi.org/10.1080/09712119.2017.1394860

El-Mandrawy, S. A. M., y Farag, G. K. (2017). Molecular Characterization, Hematological and Biochemical Studies on Foot and Mouth Disease Virus Serotype O in Buffaloes and Cows in Dakahlia Governorate, Egypt. Zagazig Veterinary Journal, 45(2), 156-164. https://doi.org/10.21608/ zvjz.2017.7888

Gamietea, I. J. (2019). Ganadería bovina en el área de influencia de la EEA San Pedro. Recomendaciones sobre sanidad, nutrición y manejo. AER San Pedro, INTA. Recuperado de: https://repositorio.inta.gob.ar/handle/20.500.12123/6352

Gelsinger, S. L., Smith, A. M., Jones, C. M., y Heinrichs, A. J. (2015). Comparison of radial immunodiffusion and ELISA for quantification of bovine immunoglobulin $\mathrm{G}$ in colostrum and plasma. Journal of dairy science, 98(6), 4084-4089. https://doi.org/10.3168/jds.2014-8491

Godden, S. M., Lombard, J. E., y Woolums, A. R. (2019). Colostrum management for dairy calves. Veterinary Clinics: Food Animal Practice, 35(3), 535-556. https://doi.org/10.1016/j. cvfa.2019.07.005

Guerra, M. G., Veras, A. S. C., Santos, V. L. F., Ferreira, M. A., Novaes, L. P., Barreto, L. M. G., ... y Silva, L. R. (2018). Protein metabolic profile of dairy cows fed corn and urea pasture. Arquivo Brasileiro de Medicina Veterinária e Zootecnia, 70(4), 1266-1274. https://doi. org/10.1590/1678-4162-9677 
Guzmán, V., y Olivera-Angel, M. (2020). 2. Calostrogénesis, digestión y absorción del calostro. Fondo Editorial Biogénesis, 17-30. Recuperado de https://revistas.udea.edu.co/index.php/ biogenesis/article/view/342145/20802566

Hampe, M., y Wehrend, A. (2019). Bestimmung der Immunglobulin-G-Versorgung beim neugeborenen Kalb. Tierärztliche Praxis Ausgabe G: Großtiere / Nutztiere, 47(2), 97-109. https://doi. org/10.1055/a-0858-4038

Holdridge, L.R. 1978. Ecología basada en zonas de vida. Instituto Interamericano de Cooperación para la Agricultura (IICA): Serie de libros y materiales educativos No. 34.

Ježek, J., Malovrh, T., y Klinkon, M. (2012). Serum immunoglobulin (IgG, IgM, IgA) concentration in cows and their calves. Acta Agriculturae Slovenica, 100 (Suppl. 3), 295-298. Recuperado de: http://aas.bf.uni-lj.si/zootehnika/supl/3-2012/ PDF/3-2012-295-298.pdf

Kaneko, Jiro J., Harvey, J. W., y Bruss, M. (2008). Clinical biochemistry of domestic animals (6a ed.). San Diego: Elsevier/Academic Press.

Klinkon, M., y Ježek, J. (2012). Values of Blood Variables in Calves. A Bird's-Eye View of Veterinary Medicine, 301-320. https://doi. org/10.5772/32100

Lago, A., Socha, M., Geiger, A., Cook, D., Silvadel-Río, N., Blanc, C., ... y Leonardi, C. (2018). Efficacy of colostrum replacer versus maternal colostrum on immunological status, health, and growth of preweaned dairy calves. Journal of dairy science, 101(2), 1344-1354. https://doi. org/10.3168/jds.2017-13032

Lawrence, K., Broerse, N., Hine, L., Yapura, J., y TuIley, W. J. (2017). Prevalence of failure of passive transfer of maternal antibodies in dairy calves in the Manawatu region of New Zealand. New Zealand Veterinary Journal, 65(1), 1-5. https://doi. org/10.1080/00480169.2016.1224207

Mulder, R., Fosgate, G. T., Tshuma, T., y Lourens, D. C. (2018). The effect of cow-level factors on colostrum quality, passive immunity and health of neonatal calves in a pasture-based dairy operation. Animal Production Science, 58(7), 12251232. https://doi.org/10.1071/AN16689

Pardo M, D., y Oliver E, O. (2012). Identificación de agentes infecciosos asociados con Diarrea Neonatal Bovina en la Sabana de Bogotá. MVZ Córdoba,
17(3), 3162-3168. Recuperado de https://www. redalyc.org/articulo.oa?id $=69325096010$

Petzl, W., Zerbe, H., Günther, J., Seyfert, H. M., Hussen, J., y Schuberth, H.-J. (2018). Pathogenspecific responses in the bovine udder. Models and immunoprophylactic concepts. Research in Veterinary Science, 116, 55-61. https://doi. org/10.1016/j.rvsc.2017.12.012

Roa-Vega, M. L., Ladino-Romero, E. A., y Hernández-Martínez, M. C. (2017). Indicadores de bioquímica sanguínea en bovinos suplementados con Cratylia argentea y Saccharomyces cerevisiae. Pastos y Forrajes, 40(2), 144-151. Recuperado de http://scielo.sld.cu/pdf/pyf/v40n2/ pyf08217.pdf

Sears, K. T., Tennant, S. M., Reymann, M. K., Simon, R., Konstantopoulos, N., Blackwelder, W. C., ... y Pasetti, M. F. (2017). Bioactive immune components of anti-diarrheagenic enterotoxigenic Escherichia coli hyperimmune bovine colostrum products. Clin. Vaccine Immunol., 24(8), e00186-16. https://doi.10.1128/CVI.00186-16

Shawky, S. M., Thabet, N. S., Orabi, S. H., y Nayel, M. A. (2016). A Comparative Study on the Hemato-Biochemical and Immunological Effects of the Hexavalent FMD Vaccine Alone or in Combination with Trivalent FMD Vaccine in Cattle. Journal of Biosciences and Medicines, 4(1), 16-26. https:// doi.org/10.4236/jbm.2016.41003

Sherwin, G., y Down, P. (2018). Calf immunology and the role of vaccinations in dairy calves. In Practice, 40(3), 102-114. http://dx.doi. org/10.1136/inp.k952

Sordillo, L. M., Contreras, G. A., \& Aitken, S. L. (2009). Metabolic factors affecting the inflammatory response of periparturient dairy cows. Animal health research reviews, 10(1), 53. https:// doi.org/10.1017/S1466252309990016

Soriano, M. F. (2014). Evaluación de la efectividad de la vacuna Escherichia Coli k99 en bovinos de leche en dos fincas en la sabana de Bogotá (Tesis de Pregrado). Universidad de la Salle, Bogotá. Recuperado de https://ciencia.lasalle.edu.co/cgi/ viewcontent.cgi?article $=1005 \&$ context $=$ medici na_veterinaria

Stark, A., Wellnitz, O., Dechow, C., Bruckmaier, R., y Baumrucker, C. (2015). Colostrogenesis during an induced lactation in dairy cattle. Journal of Animal Physiology and Animal Nutrition, 99(2), 356-366. https://doi.org/10.1111/jpn.12205 
Tizard, I., y Payne, S. L. (2019). Active Immunization. Recuperado el 20 de mayo de 2019, de https://www.msdvetmanual.com/pharmacology/ vaccines-and-immunotherapy/active-immunization

Tóthová, C., Mihajlovičová, X., y Nagy, O. (2017). The Use of Serum Proteins in the Laboratory Diagnosis of Health Disorders in Ruminants. En The Husbandry, Economic and Health Aspects (pp. 125-126). IntechOpen. https://doi. org/10.5772/intechopen.72154

Uetake, K., Akiyama, K., y Tanaka, T. (2014). Relationship between stress levels of the antepartum cow and her newborn calf. Animal Science Journal, 85(1), 81-84. https://doi.org/10.1111/ asj.12078
Yepes, M., y Prieto, C. (2011). Relación de la concentración de proteína sérica, la calidad de calostro y la ganancia de peso en terneros lactantes en hatos de la sabana de Bogotá (Tesis de Pregrado). Universidad de la Salle, Bogotá. Recuperado de https://ciencia.lasalle.edu.co/cgi/viewcontent.cgi ?article $=1111 \&$ context=zootecnia

Windeyer, M. C., y Gamsjäger, L. (2019). Vaccinating Calves in the Face of Maternal Antibodies: Challenges and Opportunities. Veterinary Clinics: Food Animal Practice, 35(3), 557-573. https://doi.org/10.1016/j.cvfa.2019.07.004 
